\title{
Atypical presentation of a gastric stromal tumor masquerading as a giant intraabdominal cyst: A case report
}

\author{
KE-KANG SUN ${ }^{1}$, SONG XU ${ }^{2}$, JINZHEN CHEN ${ }^{2}$, GANG LIU ${ }^{1}$, XIAOJUN SHEN ${ }^{1}$ and XIAOYANG WU ${ }^{1}$ \\ Departments of ${ }^{1}$ Gastrointestinal Surgery and ${ }^{2}$ Pathology, Kunshan First People's Hospital Affiliated to Jiangsu University, \\ Kunshan, Jiangsu 215300, P.R. China
}

Received April 13, 2015; Accepted July 12, 2016

DOI: $10.3892 / \mathrm{ol} .2016 .4968$

\begin{abstract}
Gastrointestinal stromal tumors (GISTs) are mesenchymal neoplasms that arise in the gastrointestinal tract, accounting for $\sim 1 \%$ of gastric malignancies. The present study reports the case of a GIST of the stomach in a 75-year-old man who presented with abdominal distension and anorexia for 1 month. Gastroscopy was unremarkable. Ultrasound and computed tomography (CT) scans showed a giant intraabdominal cystic lesion of unknown origin. The lesion was initially believed to be a duplication cyst, a pancreatic pseudocyst or a liver cyst in the pre-operative diagnosis. Exploratory laparotomy revealed a cystic lesion of the lesser sac originating from the lesser curvature of the stomach. A distal gastrectomy with en bloc resection of the lesion was performed. The intraoperative frozen section showed a spindle-cell GIST and microscopically negative margins. The patient was treated with imatinib for 1 year. The latest CT scan at 14 months post-surgery did not show any recurrence. Although GISTs presenting as predominantly cystic lesions are very rare, they should be considered in the differential diagnosis of cystic lesions of the upper abdomen.
\end{abstract}

\section{Introduction}

Gastrointestinal stromal tumors (GISTs) are the most frequently occurring mesenchymal neoplasms of the gastrointestinal tract (1). The crude annual incidence of GISTs varies across the globe, although the incidence is $\sim 130$ cases per 1 million individuals in a population (2). In total, $50-70 \%$ of the tumors originate in the stomach, while $20-30 \%$ arise in the small bowel, with the duodenum being the rarest site $(1,3)$. Contrast-enhanced computed tomography (CT) is the imaging method of choice to characterize an abdominal mass, to

Correspondence to: Mr. Xiaoyang Wu, Department of Gastrointestinal Surgery, Kunshan First People's Hospital Affiliated to Jiangsu University, 91 Qianjin Road, Kunshan, Jiangsu 215300, P.R. China

E-mail: fsyx7skk@163.com

Key words: gastrointestinal stromal tumors, intraabdominal cyst evaluate its extent, and to determine the presence or absence of metastatic disease (4). The vast majority of GISTs are solid tumors, and cystic change is uncommon $(5,6)$. There is no significant difference between cystic and solid GISTs in terms of the treatment and prognosis. For peritoneal cystic lesions, the diagnosis of GIST is not initially considered. The 5-year survival rate of patients with GIST is $70 \%$ (7).

The most common intraabdominal cystic lesion originates from the mesentery or retroperitoneal space (8). Other cystic lesion include duplication cysts, pancreas pseudocysts or liver cysts. The present study reports the case of a large cystic lesion arising from the stomach, which was ultimately diagnosed as a GIST.

\section{Case report}

A 75-year-old man presented to Kunshan First People's Hospital Affiliated to Jiangsu University (Kunshan, Jiangsu, China) on February 25, 2015 with abdominal distension and anorexia that had persisted for 1 month. There was no history suggestive of gastric outlet obstruction, and no vomiting, melena or constitutional symptoms. The patient had undergone no past surgeries and had no history of pancreatitis. Upon examination, no abdominal mass was palpable. A CT scan of the abdomen was performed, which showed a well-defined exophytic cystic lesion located between the liver and the stomach (Fig. 1). No metastatic localization was detectable in the liver or lungs, and neither peritoneal dissemination nor lymph node spreading were revealed. Gastroscopy observed a large bulge at the lesser curvature of the stomach, with an unremarkable mucosa (Fig. 2A). Tumor markers, including carcinoembryonic antigen, $\alpha$-fetoprotein, cancer antigen (CA)125 and CA19-9, were all within the normal ranges. A pre-operative diagnosis of a liver cyst, a pancreas pseudocyst or a duplication cyst was formed.

Laparotomy revealed a reddish, bulky and vascularized mass arising from the lesser curvature of the stomach. A distal gastrectomy with en bloc resection of the mass was performed (Fig. 2B), and the continuity of the gastrointestinal tract was restored by a gastroduodenal anastomosis (Billroth I). For histopathological analysis, tissue sections $(4 \mathrm{~mm})$ were incubated with $4 \%$ paraformaldehyde for $15 \mathrm{~min}$ at room temperature, and washed twice with Tris-buffered saline (TBS) containing $0.1 \%$ saponin. Subsequently, the tissues were incubated with 

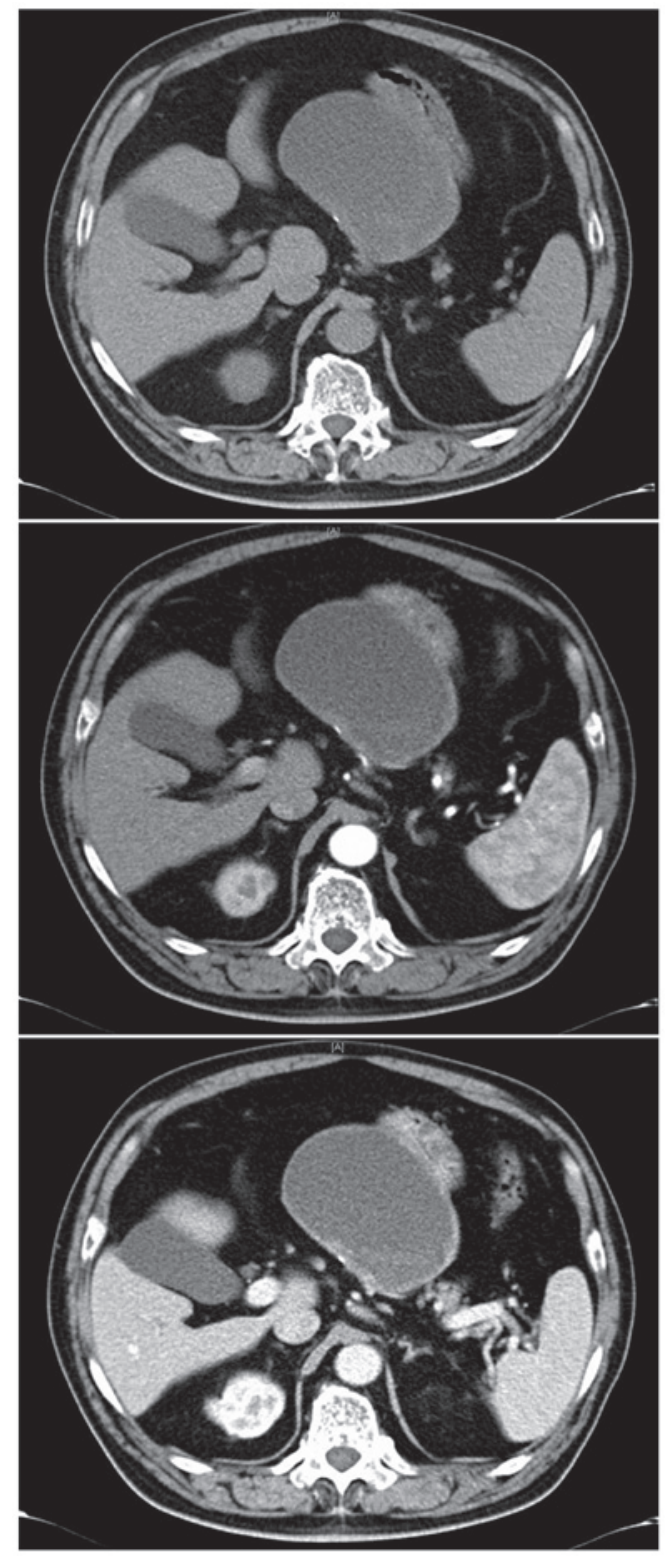

Figure 1. Computed tomography scans revealing a giant intraabdominal cystic lesion in the lesser sac, with clear boundaries.

TBS containing $0.3 \% \mathrm{H}_{2} \mathrm{O}_{2}, 0.1 \%$ saponin and $0.02 \% \mathrm{NaN}_{3}$ for $30 \mathrm{~min}$ to bock endogenous peroxidase activity, stained with hematoxylin and eosin and observed under an optical microscope. The histopathological analysis showed that the tumor was composed of spindle cells arranged in bundles and braids. The diagnosis was of a GIST with microscopically negative margins.

The macroscopically solid tumoral lesion measured $13 \times 10 \times 6 \mathrm{~cm}$. Routine pathology confirmed a spindle-cell GIST (Fig. 2B) and necrotic cystic degeneration. Tumor sections were stained with mouse anti-c-kit [cluster of differentiation (CD)117] polyclonal antibody (cat. no. sc-393910), mouse anti-CD34 monoclonal antibody (cat. no. sc-19621), mouse anti-desmin monoclonal antibody (cat. no. sc-23879) and mouse anti-smooth muscle actin monoclonal antibody (cat. no. sc-53015; all 1:500; all Santa Cruz Biotechnology, Inc., Dallas, TX, USA), followed by horseradish peroxidase-conjugated goat anti-mouse secondary antibody (cat. no. sc-2005;
A

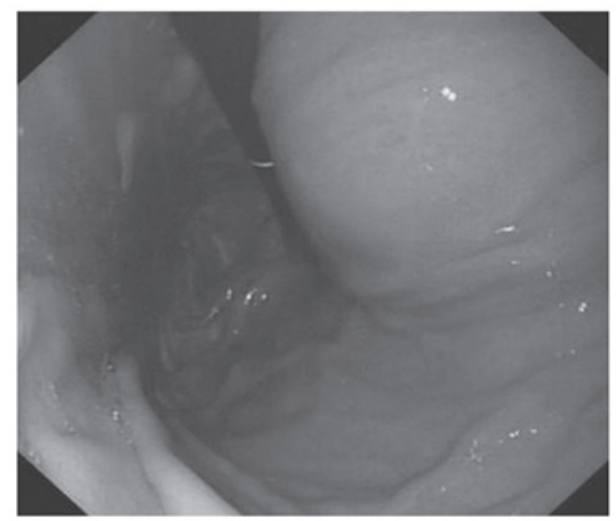

B

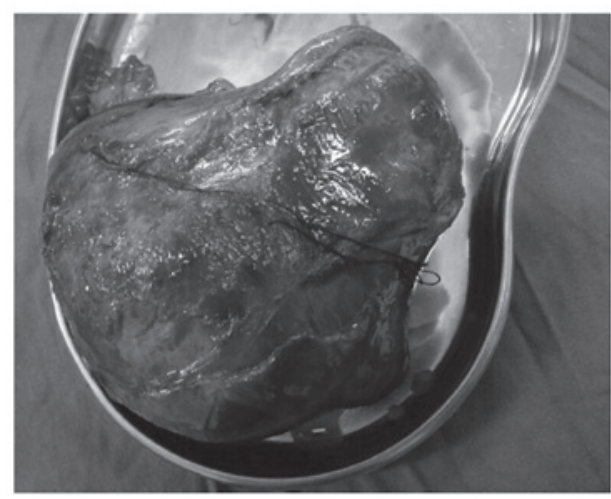

Figure 2. (A) Endoscopic view of the stomach showing a large bulge at the lesser curvature. (B) Intraoperative view of the tumor.

1:1,000; Santa Cruz Biotechnology, Inc.). The tumor lesion showed strong positive staining for c-kit and CD34, whereas $\mathrm{S}-100$, desmin and smooth muscle actin were negative. The mitotic count was $<5 / 50$ high-power fields. The lymph nodes that were removed were uninvolved. The patient made an uneventful post-operative recovery, being discharged from hospital at 10 days post-surgery. The patient remained under follow-up by the hospital's medical oncology service and was administered $400 \mathrm{mg}$ imatinib daily for 1 year prior to abandoning imatinib therapy for economic reasons. The latest CT scan, performed at 14 months post-surgery, did not show any signs of recurrence.

\section{Discussion}

GISTs are the most common sarcomas of the gastrointestinal tract, accounting for $\sim 1 \%$ of gastric neoplasms (9). It is difficult to predict the biological behavior of a GIST, which ranges from benign to malignant, and the tumor size and mitotic index are believed to be the most reliable prognostic factors (10). The vast majority of GISTs are solid tumors and the minority of lesions present with cystic change upon rapid tumor growth and necrosis (11). GISTs presenting with complete cystic change and as an extragastric lesion is extremely rare.

Due to the rarity and different modes of presentation of GISTs, the pre-operative diagnosis is extremely difficult. A total of $25 \%$ of GISTs are found incidentally during imaging or surgery for other disorders (12). Instrumental examination, including gastroscopy, CT scan and endoscopic ultrasonography permits mesenchymal tumors to be distinguished from 
other tumor types. Fine-needle tissue acquisition biopsy samples obtained through endoscopy or endoscopic ultrasonography cannot be used for a reliable proliferation assessment as they are too small for immunohistochemical analyses (13). An accurate diagnosis of a GIST is often formed only after surgery.

In conclusion, the vast majority of GISTs are solid tumors, and cystic change is uncommon. Preoperatively, an intraabdominal cyst may be considered a duplication cyst, a pancreatic pseudocyst or a liver cyst. To the best of our knowledge, the present study is the first to report a case of GIST presenting as a cystic lesion. The patient presented with a cystic lesion originating from the stomach, which was diagnosed as GIST via a histological analysis. Therefore, GISTs presenting as cystic lesions, although rare, should be considered in the differential diagnosis of cystic lesions of the upper abdomen.

\section{References}

1. Joensuu H, Hohenberger P and Corless CL: Gastrointestinal stromal tumor. Lancet 382: 973-983, 2013.

2. Nilsson B, Bümming P, Meis-Kindblom JM, Odén A, Dortok A, Gustavsson B, Sablinska K and Kindblom LG: Gastrointestinal stromal tumors: The incidence, prevalence, clinical course, and prognostication in the preimatinib mesylate era - a population-based study in western Sweden. Cancer 103: 821-829, 2005.

3. Samelis GF, Ekmektzoglou KA and Zografos GC: Gastrointestinal stromal tumours: Clinical overview, surgery and recent advances in imatinib mesylate therapy. Eur J Surg Oncol 33: 942-950, 2007.

4. Abdel-Monem S, Enaba MM, Hassan TA, et al: Multislice CT imaging of gastrointestinal stromal tumors (GISTs). Egypt J Radiol Nuc Med 42: 1-7, 2011.
5. Kumar A, Jakhmola CK, Chauhan SS and Singh A: A typical presentation of gastrointestinal stromal tumor masquerading as a large duodenal cyst: A case report. Int J Surg Case Rep 9: 123-126, 2015.

6. Cavallaro G, Sadighi A, Polistena A, Rossi V, Cristaldi M, Paparelli C and De Toma G: Pedunculated giant GISTs of the stomach with exophytic growth: Report of two cases. Int J Surg 6: e80-e82, 2008.

7. Joensuu H, Vehtari A, Riihimäki J, Nishida T, Steigen SE, Brabec P, Plank L, Nilsson B, Cirilli C, Braconi C, et al: Risk of recurrence of gastrointestinal stromal tumour after surgery: An analysis of pooled population-based cohorts. Lancet Oncol 13: 265-274, 2012.

8. Choi BI, Kim YH and Han JK: Hepatobiliary and pancreatic: Cystic liver lesion in a man with abdominal pain. J Gastroenterol Hepatol 14: 609-613, 1999.

9. Goettsch WG, Bos SD, Breekveldt-Postma N, Casparie M, Herings RM and Hogendoorn PC: Incidence of gastrointestinal stromal tumours is underestimated: Results of a nation-wide study. Eur J Cancer 41: 2868-2872, 2005.

10. Bucher P, Egger JF, Gervaz P, Ris F, Weintraub D, Villiger P, Buhler LH and Morel P: An audit of surgical management of gastrointestinal stromal tumours (GIST). Eur J Surg Oncol 32: 310-314, 2006.

11. Shaikh ST, Upwanshi MH, Shetty TS, Ghetla SR and Gheewala H: A Large Cystic Variant of Gastro-intestinal Stromal Tumour arising from the Jejunum: A Case Report. J Clin Diagn Res 9: $11-12,2015$

12. Al-Thani H, El-Menyar A, Rasul KI, Al-Sulaiti M, El-Mabrok J, Hajaji K, Elgohary $\mathrm{H}$ and Tabeb A: Clinical presentation, management and outcomes of gastrointestinal stromal tumors. Int J Surg 12: 1127-1133, 2014.

13. Ricci R, Chiarello G, Attili F, Fuccio L, Alfieri S, Persiani R, Di Pietro S, Martini M, Costamagna G, Larocca LM and Larghi A: Endoscopic ultrasound-guided fine needle tissue acquisition biopsy samples do not allow a reliable proliferation assessment of gastrointestinal stromal tumours. Dig Liver Dis 47: 291-295, 2015. 